\title{
Autosomal dominant neovascular inflammatory vitreoretinopathy — a case series
}

\author{
Małgorzata Kowalczyk, Robert Rejdak@ \\ Department of General Ophthalmology and Pediatric Ophthalmology Service, Medical University in Lublin, Lublin, Poland
}

\begin{abstract}
The objective of our study was to report the course of the disease in a family affected with autosomal dominant neovascular inflammatory vitreoretinopathy (ADNIV, OMIM\#193235). ADNIV is a very rare inherited blinding disease due to mutations in CAPN5 gene.

We assembled a retrospective observational case series of ADNIV patients. We noticed first symptoms in different ages, similar course of the disease and its progression leading in most cases to complete blindness despite treatment.
\end{abstract}

KEY WORDS: autosomal dominant neovascular inflammatory vitreoretinopathy; ADNIV; uveitis; neovascularization; proliferative vitreoretinopathy; cystoid macular edema; CAPN5; calpain-5

Ophthalmol J 2019; Vol. 4, 31-39

\section{INTRODUCTION}

Autosomal dominant neovascular inflammatory vitreoretinopathy (ADNIV) is an autoimmune uveitis belonging to inherited vitreoretinal dystrophies [1]. It is caused by the mutations in CAPN5 gene (calpain-5) located on chromosome 11q13 [2,3]. The first scientific work describing ADNIV was published in 1990 by Steven Bennett and co-workers [4]. Autosomal dominant neovascular inflammatory vitreoretinopathy occurs with a frequency of approximately one in 1000000 births and has been found worldwide [5].

The first symptoms can appear at all ages [5]. Autosomal dominant neovascular inflammatory vitreoretinopathy is characterized by non-specific uveitis in the anterior chamber and vitreous leading to panuveitis, iris and retinal neovascularization, cystoid macular edema, abnormal retinal pigmentation, vitreous hemorrhage, intraocular fibrosis with membrane formation and tractional retinal detachment. Sometimes we observe the development of secondary glaucoma. It is due to uveitic glaucoma, steroid-induced glaucoma or angle closure glaucoma. The hypotony is also observed in ADNIV patients [6].
Unfortunately, despite treatment the disease is very progressive and often leads to complete blindness and, subsequently, phthisis. Another characteristic sign of ADNIV is loss of the b-wave amplitude and photoreceptors' degeneration on electroretinography (ERG) recordings [3, 7].

\section{CASE REPORT}

A retrospective case series was assembled from the medical reports of ADNIV patients from 1988 to present (2019). We reviewed the course of illness in 7 of 20 members of a four-generation family affected by ADNIV disease -2 sisters (Patient $1-\mathrm{P} 1$, Patient $2-\mathrm{P} 2$ ), their father (Patient 3 - P3), father's two sisters (Patient $4-\mathrm{P} 4, \mathrm{~Pa}-$ tient 5-P5), Patient's 5 daughter (Patient 6-P6) and father's brother (Patient 7 - P7) (Fig. 1). All patients provided informed consent for taking part in this retrospective study. They were referred to our Department due to the suspicious of family vitreoretinopathy. Initially the diagnosis was based just on a typical clinical history and plotted pedigree by geneticist. The patients underwent a complete 


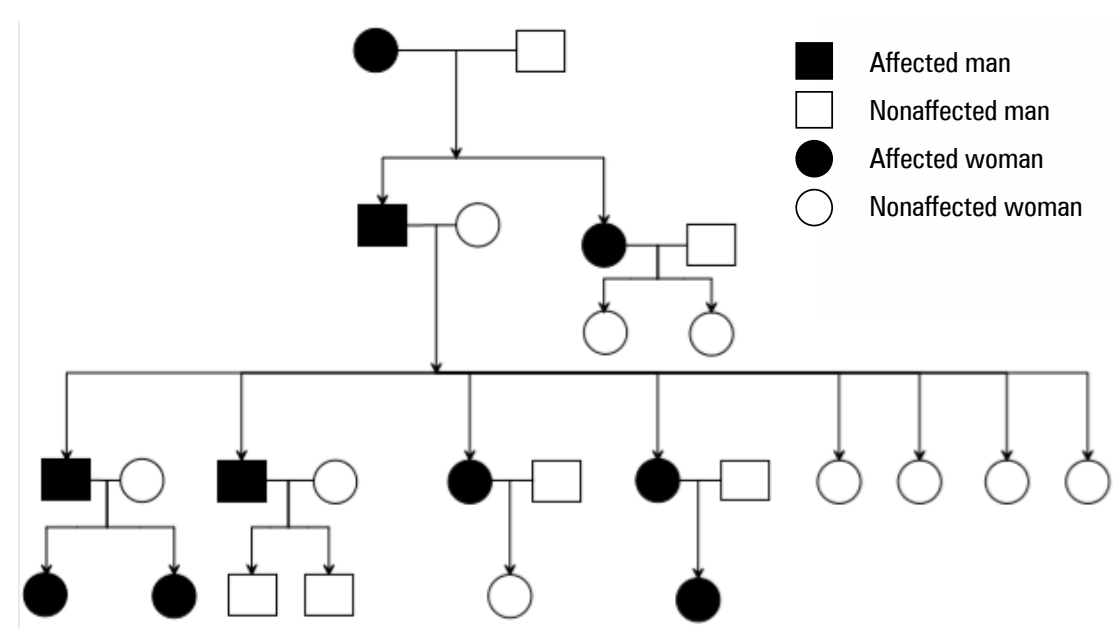

FIGURE 1. Family tree — affected and nonaffected members of the family

ophthalmological evaluation. Visual acuity assessment, slit lamp biomicroscopy and fundoscopy were performed. The image of macula was observed using the optical coherence tomography (OCT) and angioOCT. The fundus photographs were recorded. The patients were diagnosed using ERG testing (P1, $\mathrm{P} 2)$ and were referred for genetic evaluation (P1, $\mathrm{P} 2)$. The mutations in exon 6 of the CAPN5 gene were not found but this does not exclude the presence of ADNIV, because there are other not examined fragments of CAPN5 gene.

\section{PATIENT 1}

A 20-year-old woman with ADNIV has been followed up in our outpatient clinic since 2008. She was referred due to previous uveitis and gradual deterioration of visual acuity which started when she was 9. On examination, her best corrected visual acuity (BCVA) was 0.4 in the right eye $(\mathrm{OD}-\mathrm{ocu}$ lus dextra) and 0.6 in the left eye (OS - oculus sinistra). Her anterior segment examination was normal in both eyes. We noticed inflammatory cells in the vitreous in both eyes. The posterior segment examination showed normal picture of optic discs and narrowing of the arteries. With progression of the disease, inflammatory cells in the anterior segment and vitreous increased. Her uveitis was managed with topical steroids. Because of the central macula edema (CME) in both eyes the subtenon triamcinolone acetate injections were performed 2 times in her OS $(2010,2014)$ and once in her OD (2010) (Fig. 2, 3AB). The cataract started to develop in both eyes. She was referred for ERG examination where progressive damage to the function of rods and cones was determined. On the following exami- nations we noticed chronic uveitis in the vitreous and intraocular fibrosis which obscured the view of her fundus.

Genetic examination performed in 2016 raised suspicion of autosomal neovascular inflammatory vitreoretinopathy. ERG testing in 2017 confirmed the diagnosis with reduced b-wave amplitudes and photoreceptors degenerations.

On the examination performed recently, her BCVA was 0.063 in OD and 0.08 in OS. Mild anterior segment uveitis was present. On fundus examination, we observed paler optic discs, narrowing of the arteries and veins, chronic $\mathrm{CME}$ and intraocular fibrosis.

She has been also treated due to hyperthyroidism for 3 years.

\section{PATIENT 2}

A 15-year-old girl was referred to our outpatient clinic for ophthalmological evaluation in 2006. Her three years older sister (Patient 1) was treated because of uveitis of unknown etiology and deterioration of visual acuity. On the first examination when she was 4 years old her visual acuity, the anterior and posteriori segments were normal. After 2 years of follow up, her BCVA decreased to $0.7 \mathrm{OD}$ and 0.9 OS. As the disease progressed, inflammatory cells in the anterior segment and vitreous were noticed. It resulted in mild media opacity.

She was referred for genetic evaluation in 2016 where autosomal dominant neovascular inflammatory vitreoretinopathy was suspected. ERG testing in 2017 confirmed the diagnosis of ADNIV. It showed reduced b-wave amplitudes and photoreceptors degenerations. 


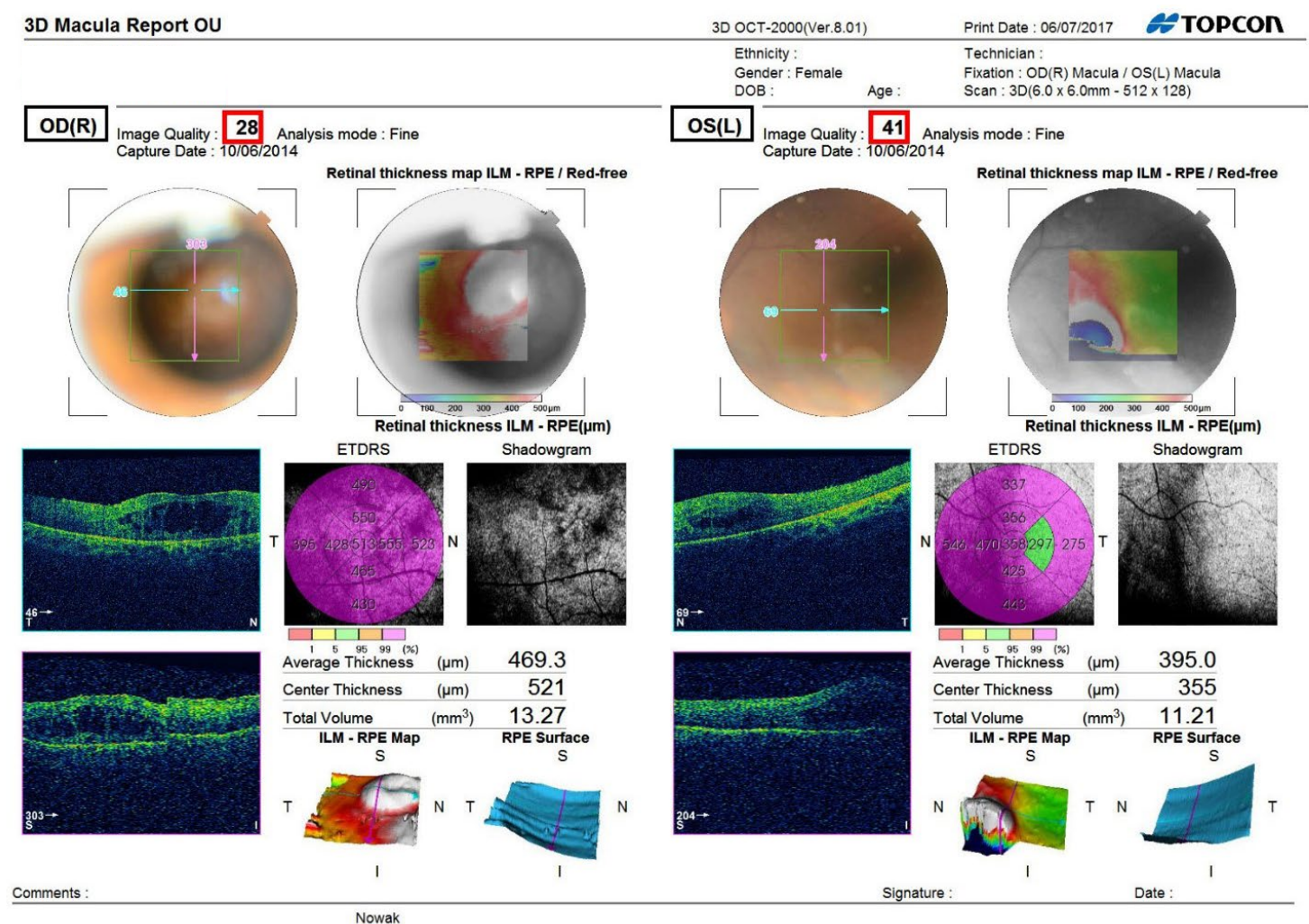

FIGURE 2. Patient 1 - cystoid macular edema in optical coherence tomography in 2014

A

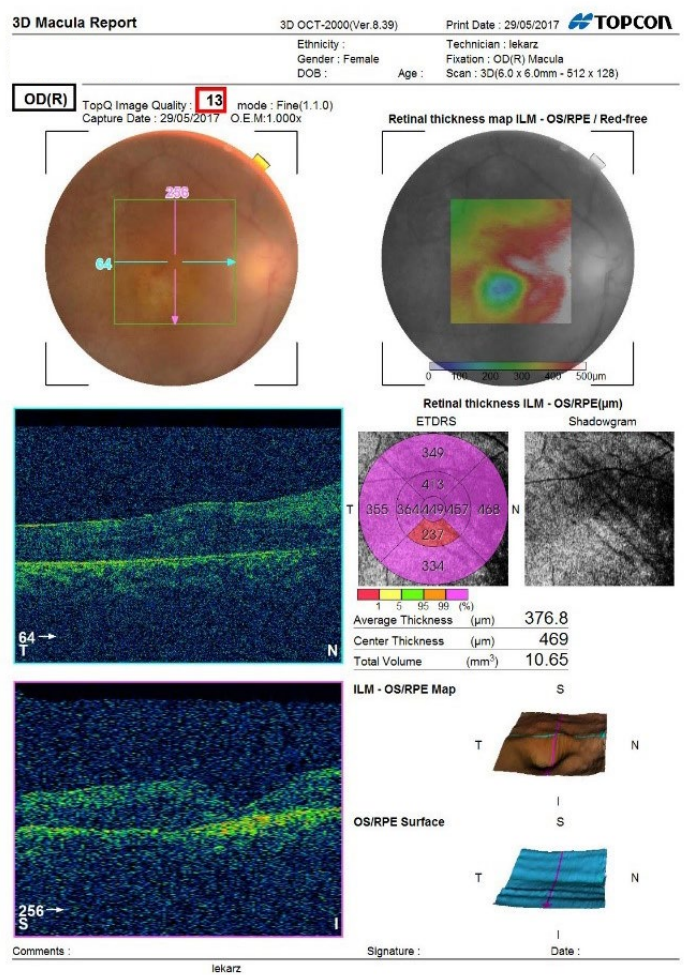

B
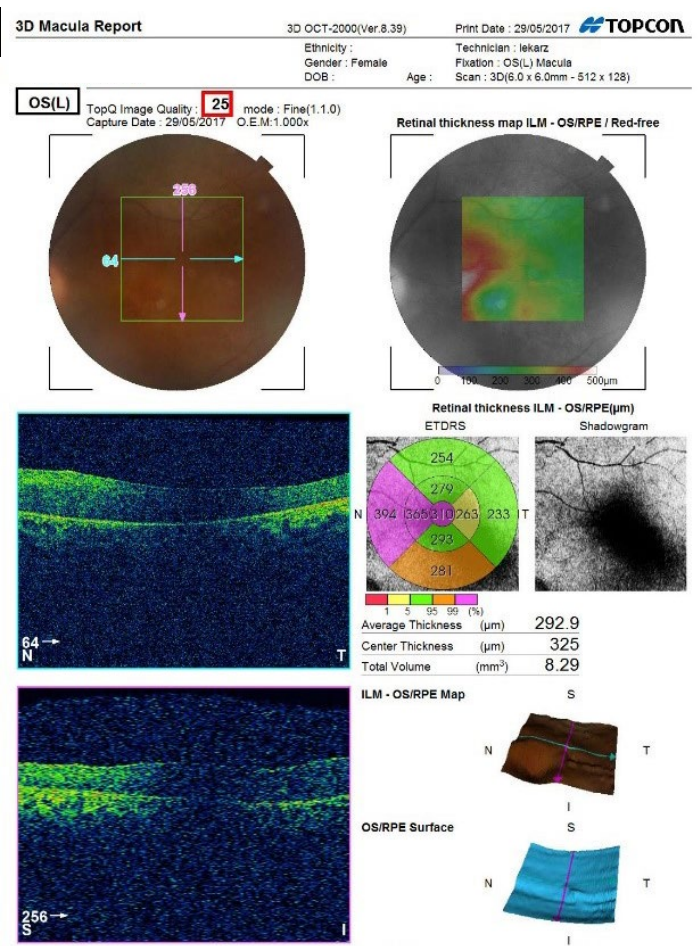

$\quad\left(\mathrm{mm}^{3}\right) \quad 8.29$

ILM-OSIRPE Map

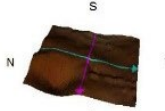

OSIRPE Surtace

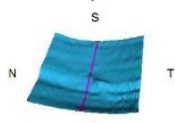

'
Date:

FIGURE $3 A B$. Patient 1 - cystoid macular edema in optical coherence tomography in the right eye (OD) and diminished central macula edema (CME) in the left eye (OS) in 2017 

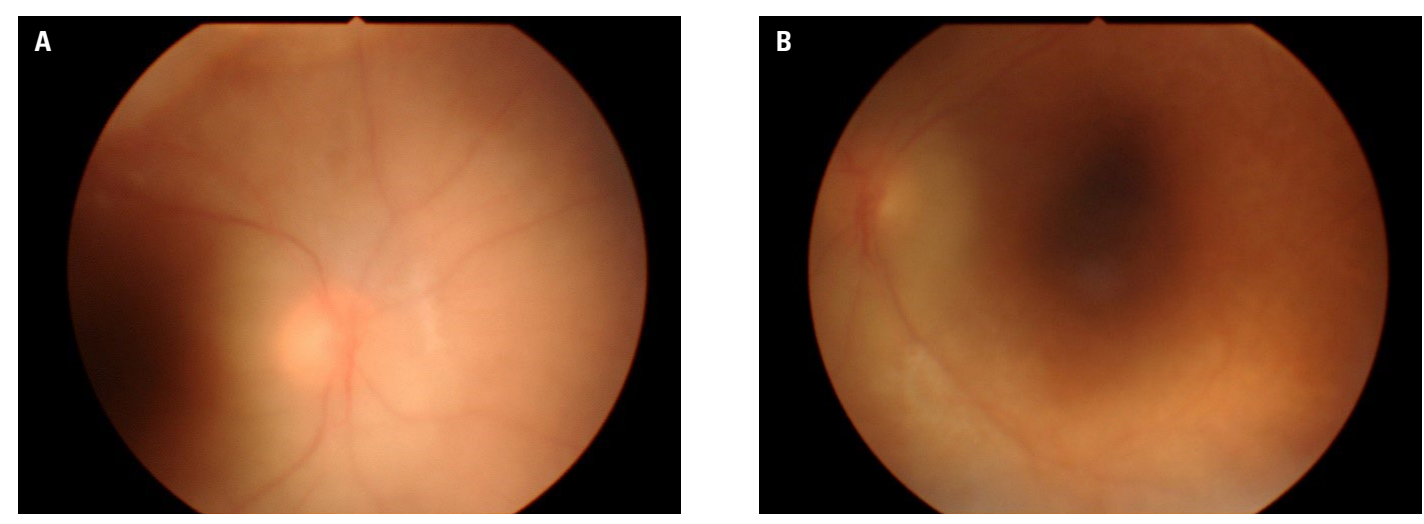

FIGURE 4AB. Patient 2 - obscured view of the fundus

A
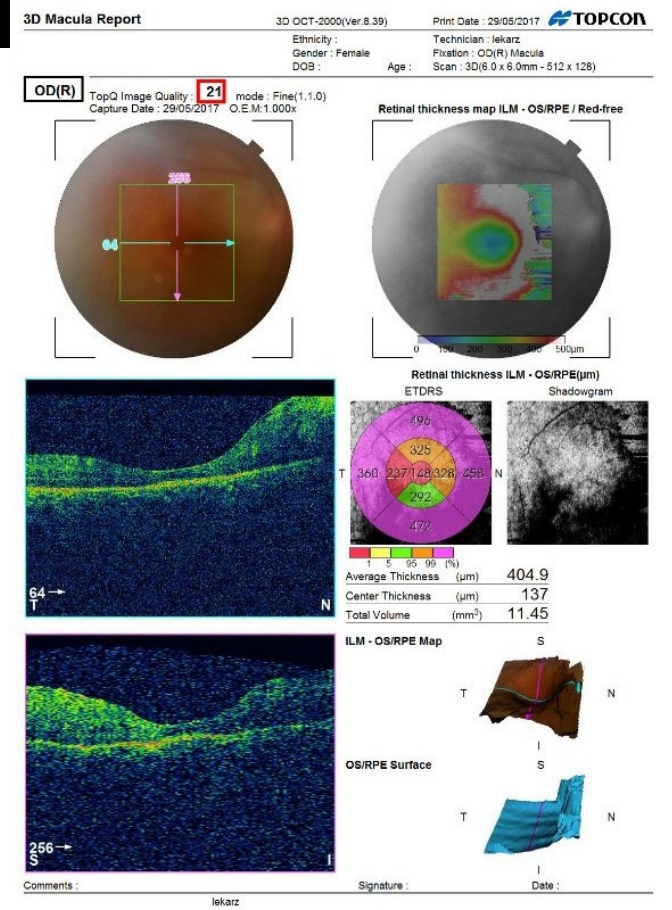

B

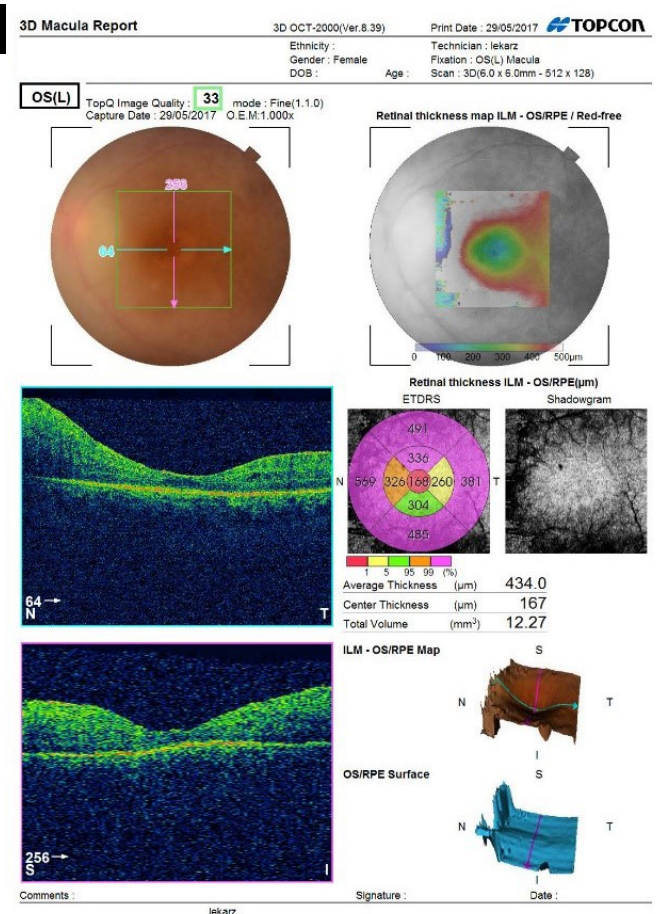

FIGURE 5AB. Patient 2 - thinning in the macula in optical coherence tomography in the right eye (OD) and the left eye (OS)

On the last examination, her BCVA was 0.7 in both eyes, the anterior segment was normal and significant uveitis in the vitreous was noticed. On her fundus examination, her optic discs were normal, whereas arteries and veins were narrowed (Fig. 4AB). The OCT examination revealed significant macular thinning in both eyes (Fig. 5AB).

She has been also treated for autoimmunological thyroiditis since 2014.

\section{PATIENT 3}

A 43-year-old man, a father of Patient 1 and 2. His first examination in our outpatient clinic was carried out in 2017. His visual acuity started to decrease when he was about 7 years old. Now he is blind. His OD vision is light perception and OS vision - no light perception. He is after cataract extraction with IOL implantation in his OD and mature cataract in his OS. The iridodonesis is visible due to subluxated lenses in both eyes. His pupils are distorted because of the posterior synechiae. Previously, he was treated due to glaucoma of both eyes. Now his eye pressure measurement was normal: $11 \mathrm{~mm} \mathrm{Hg}$ in OD and $16 \mathrm{~mm} \mathrm{Hg}$ in OS. The fundus examination was very limited because of the significant media opacity especially in OD 


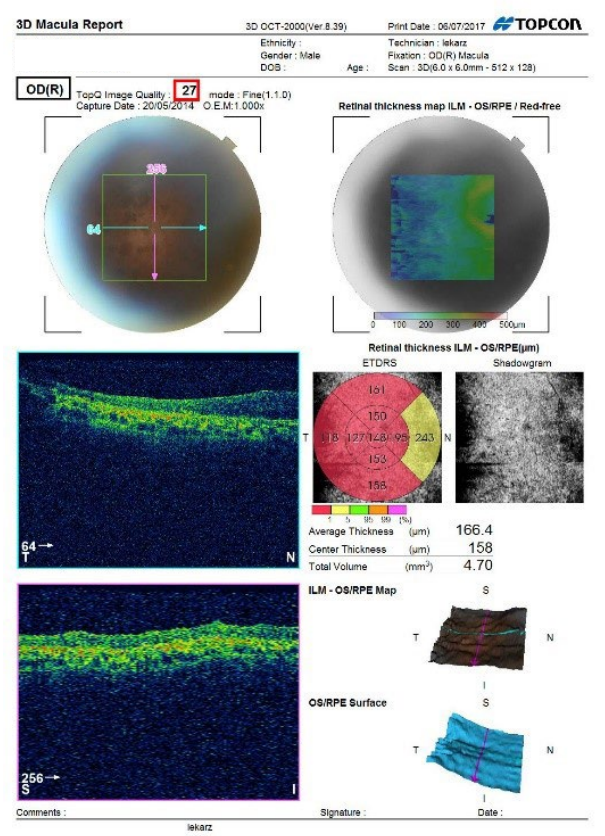

FIGURE 6. Patient 3 - thinning in the macula in optical coherence tomography

where the picture of the fundus was grey. In the OS, the optic disc was pale; very narrow arteries and veins and numerous pigment degenerations were visible (Fig. 6). Ultrabiomicroscopy B examination revealed vitreous tractions in OD (Fig. 7AB).

$\mathrm{He}$ was referred for genetic examination in 2005 together with his two sisters (Patient 4 and 5). The diagnosis of the familial inflammatory neurovitreoretinochoroidopathy was suspected.

He has been also treated for thrombocytopenia and splenomegaly.

\section{PATIENT 4}

A 45-year-old woman, a sister of Patient 3, was examined in our outpatient clinic in 2017. She is also blind, like her younger brother (Patient 3 ). Her visual acuity started to deteriorate when she was about 6 years old. In 1988, when she was 14 years old, she was diagnosed with hereditary neuropathy in both eyes. Her visual acuity was 0.1 in both eyes. It was treated frequently with intravenous steroids injections and oral steroids. Both eyes cataract was noticed in 2002. The familial neurovitreoretinochoroidopathy was suspected during genetic examination in 2005 (which she underwent together with her brother, Patient 3, and her sister, Patient 5).

Presently her visual acuity is no light perception in OD and light perception in OS. On the anterior segment examination we noticed iridodonesis due to subluxated cataract in both eyes. Grey picture of the fundus was caused by significant media opacity. Ultrabiomicrocopy B revealed intraocular fibrosis in both eyes.

She has been also treated for hypothyroidism since 2016. She underwent partially mastectomy in 2017.

\section{PATIENT 5}

A 32-year-old woman, a sister of Patient 3 and 4, was referred to our outpatient clinic in 2014 for ophthalmological examination. Her vision started to deteriorate when she was about 5 years old. In 2001, optic discs edema and uveitis in both eyes was determined. Her BCVA was 0.8 in both eyes. Her uveitis and papillitis was treated with intravenous and oral steroids. The toxoplasmosis $[\operatorname{IgM}(-), \operatorname{IgG}$ (237)] was also diagnosed and treated in this year.
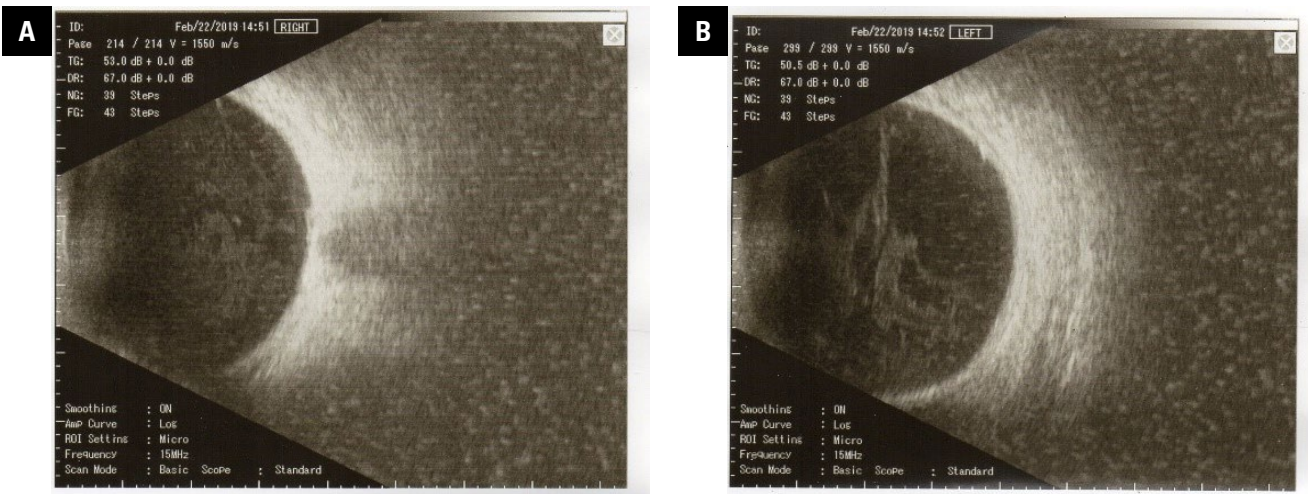

FIGURE 7AB. Patient 3 - increased density in the vitreous in the right eye (OD) and intraocular fibrosis in the left eye (OS) in ultrabiomicroscopy 


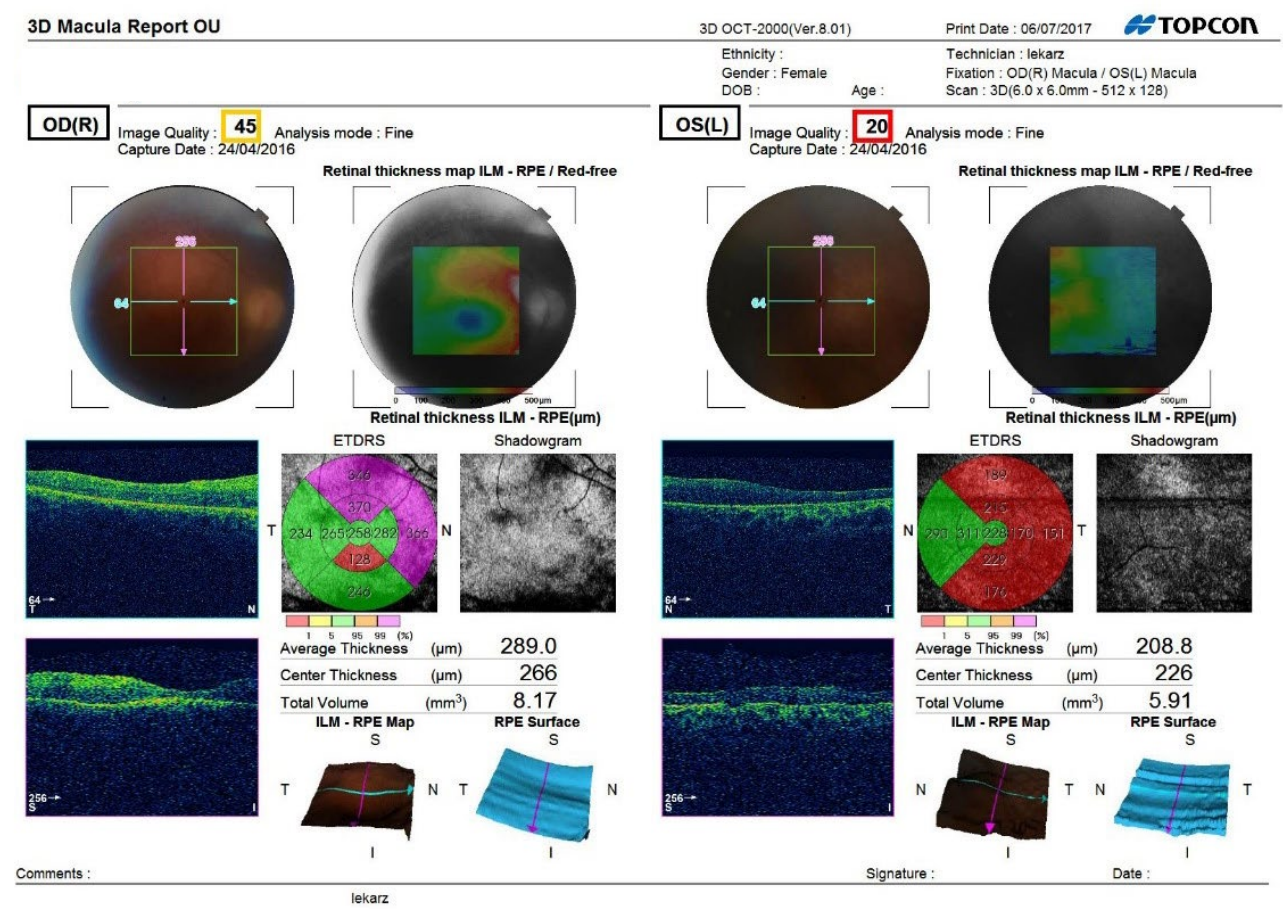

FIGURE 8. Patient 5 - diminishing of cystoid macular edema in optical coherence tomography in $0 \mathrm{U}$

She was referred for genetic examination with her brother (Patient 3) and her sister (Patient 4) and inflammatory neurovitreoretinochoroidopathy was suspected. OCT revealed CME in both eyes. It was treated with intravitreous triamcinolone acetate injections in OS (2009) and in OD (2010). The cataracts extractions were performed in both eyes in $2012 \mathrm{OD}$ and in 2013 OS. No improvement in visual acuity was obtained. With progression of the disease, reduction in cystoid macular edema was observed (Fig. 8).

On the last examination in 2017 , her visual acuity in OD was counting fingers from $30 \mathrm{~cm}$ and OS was just fingers movement before eye. On the anterior segment examination, we observed iridodonesis and on fundus examination - pale optic discs and very narrow arteries and veins. Numerous inflammatory cells were detected in the vitreous in both eyes.

\section{PATIENT 6}

A 13-year-old girl, a daughter of Patient 4, was examined in 2010 because of blurred vision in one eye. Her BCVA in OD was 1.0 and in OS was 0.6 . The anterior segment examination was normal but posterior segment revealed CME in OCT exam. It was treated with topical non-steroidal anti-inflammatory eye drops and oral acetazolamide.
It was her first and last ophthalmological examination. Unfortunately, she died in car accident when she was 14 years old.

\section{PATIENT 7}

A 37-year-old man, a brother of Patient 3, Patient 4 and 5 Patient 5 , was referred to our outpatient clinic in 2009 due to a decrease in his visual acuity in both eyes. His vision started to deteriorate at the end of the third decade of life. On the first visit the vitreoretinitis and $\mathrm{CME}$ in both eyes were diagnosed. He was treated with intravenous and oral steroids. His visual acuity was 0.3 in OD and 0.2 in OS. There was no improvement despite applied treatment. Next triamcinolone acetate injections in OS (2009) and in OD (2012) and subtenon steroid injection in OS (2010) were made. In 2012, the vitrectomy with ILM peeling in his OS was performed. Despite the treatment, the cystoid macular edema was present in OS and normal macula in OD was observed in 2015. Two years later swelling in the macula was detected in both eyes (Fig. 9AB, $10 \mathrm{AB})$. The measurement of his intraocular pressure in OD was higher $(28 \mathrm{~mm} \mathrm{Hg})$ in 2012. It was managed with topical antiglaucoma drops.

On the last examination his BCVA was 0.1 in both eyes. Anterior segment examination was normal. There was mild inflammation in the vitreous 

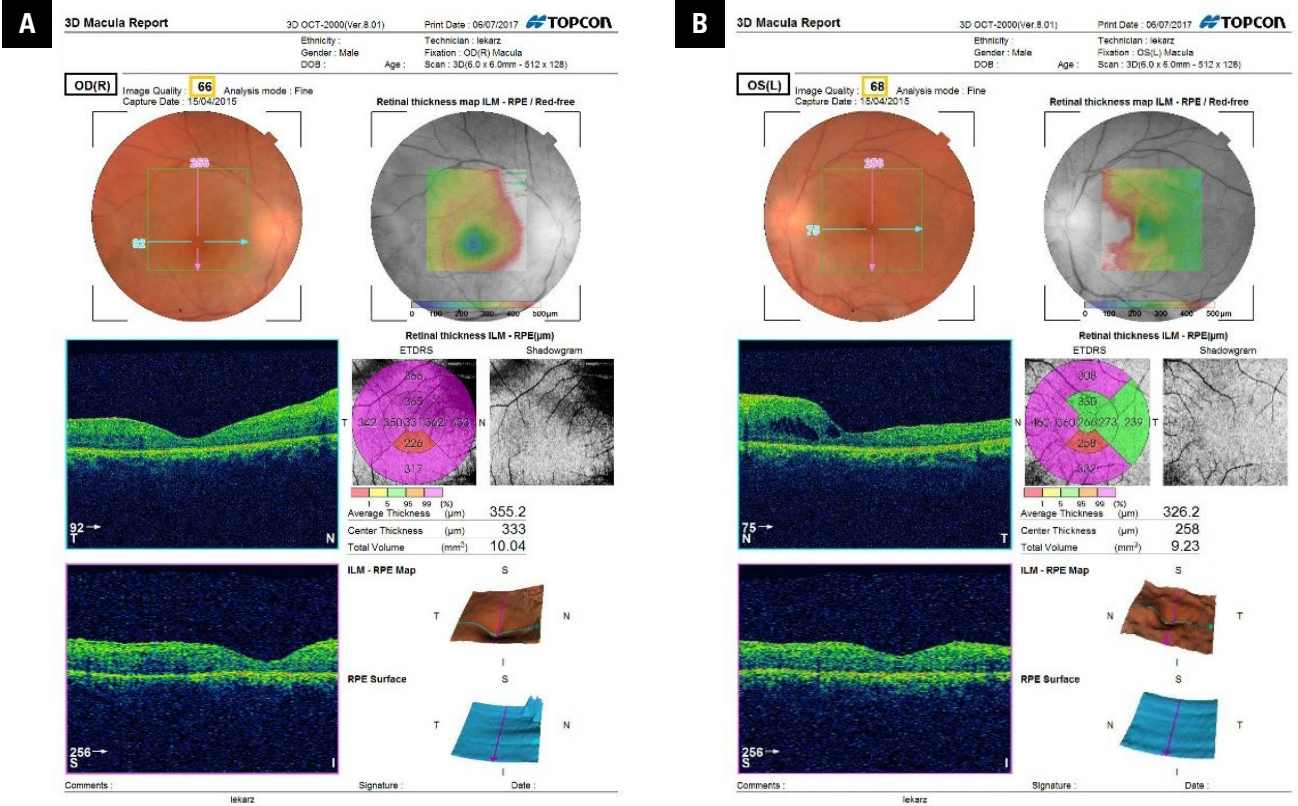

FIGURE 9AB. Patient 7 - normal macula in the right eye (OD) and cystoid macular edema in the left eye (OS) in optical coherence tomography in 2015
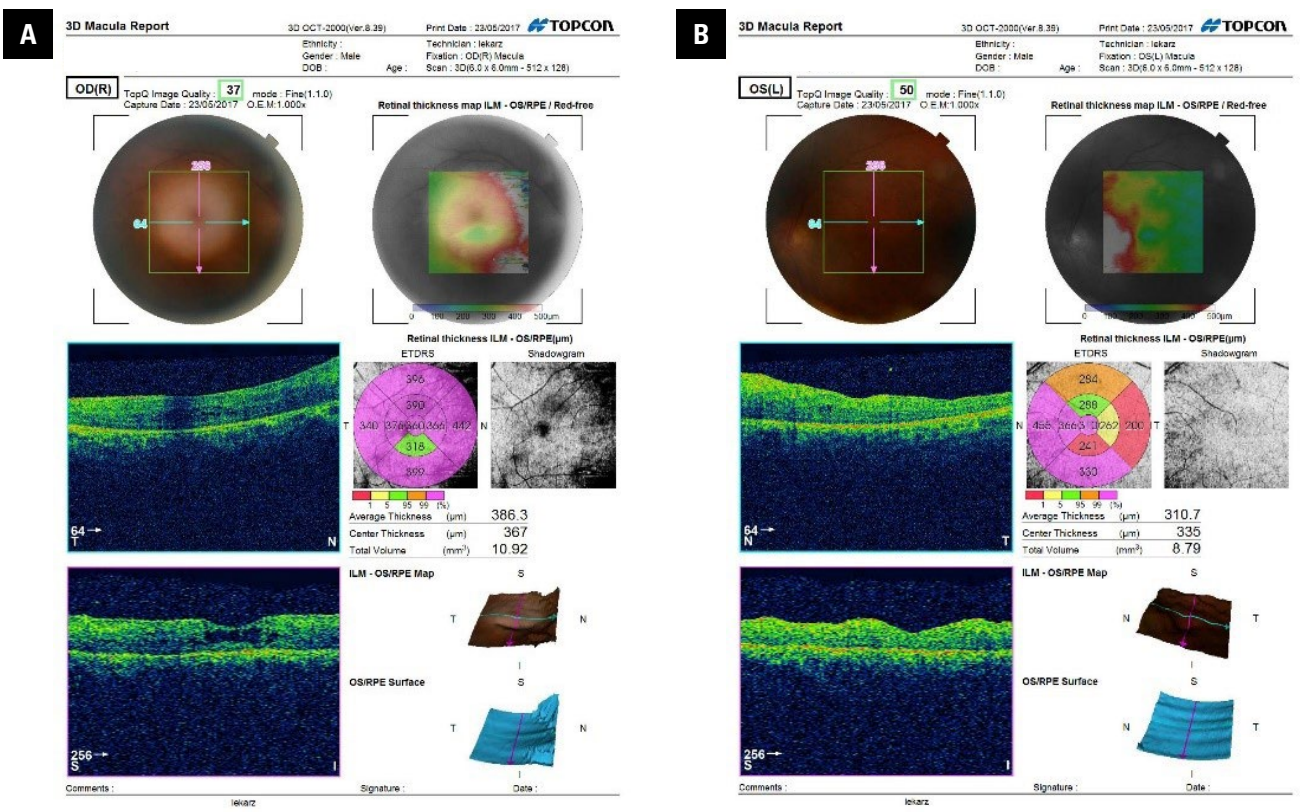

FIGURE 10AB. Patient 7 - swelling in the macula in the right eye (OD) and in the left eye (OS) in optical coherence tomography in 2017

in both eyes. In OCT exam we noticed significant thinning in the macula in both eyes. Additionally he smokes cigarettes and abuses alcohol.

\section{DISCUSSION}

Autosomal dominant neovascular inflammatory vitreoretinopathy (ADNIV) is a very serious disease leading to loss of vision. It is classified as a rare disease. The diagnosis of the illness is very difficult because its symptoms mimic much more common eye conditions like uveitis, retinitis pigmentosa, rod-cone dystrophy, proliferative vitreoretinopathy and diabetic retinopathy [4, 7-9]. A very characteristic sign of ADNIV is lack of systemic features [1]. 
The symptoms of the disease are divided into 5 stages, each lasting approximately ten years [10] The affected members of the family showed clinical signs of ADNIV disease previously reported by other authors $[2,11,12]$, including non-infectious uveitis in the anterior segment and vitreous, cystoid macular edema, epiretinal membrane formation, proliferative vitreoretinopathy, cataract, neovascular glaucoma and, ultimately, blindness. Like other authors, we observed asymmetric disease progression between the eyes of affected patient and also between affected members of the family. The clinical severity of the disease was indistinguishable among affected males and females.

The first symptoms can start at all ages [5]. In our series, first symptoms occurred in the first decade of life in all patients except Patient 7. His visual acuity started to decrease at the end of the third decade of life. We observed significant deterioration of visual acuity with age in all patients (Tab. 1). Over the years, with disease progression there were different but characteristic symptoms of ADNIV in each patient. We noticed recurrent uveitis in the anterior segment in the majority of patients and inflammation in the vitreous in all patients. The cataract formation was also observed in all patients, even in the youngest ones (Patient 1 and 2). The papilledema was noticed in three patients: Patient 3, 4 and 5. OCT detected CME in Patient 1, 6, and 7; preretinal membrane in Patient 2 and thinning in the macula in Patient 2 and 7 . We also observed raised intraocular pressure in Patient 3, 4 and 7. The examination of the anterior segment revealed the presence of iridodonesis in patients with cataract (Patient 4) and after extraction of cataract (Patient 3 and 5). All patients had more or less significant media opacity due to ongoing inflammation in the vitreous and intraocular fibrosis which especially obscured view of the fundus. Disease progression was observed in all patients. They showed the signs of stage II/III (Patient 1, 2, 6 and 7) and stage IV (Patient 3, 4 and 5) ADNIV.

On electroretinography loss of the b-wave was recorded.

In our genetic analysis (Patient 1and 2), the mutations in exon 6 of the CAPN5 gene were not detected but this does not exclude the presence of ADNIV disease because other fragments of the gene was not examined. Exon 6 of the CAPN5 gene was subjected to genetic testing because other authors [3] reported mutations in this exon.
Treatment of ADNIV poses many challenges. The pathophysiology and immunopathology are still unknown and for this reason the treatment is symptomatic rather than specific. The management of chronic immune-mediated uveitis is often very difficult and ineffective [13]. The patients received repeated injections of immunosuppressive medications to control cystoid macular edema and intraocular inflammation, but in all of them the disease progressed despite treatment. Long-term local and systemic steroid therapies are limited because of the numerous side effects. Some authors report the benefit of the fluocinolone acetonide (FA) implant in the treatment of chronic uveitis in ADNIV patients [13].

In our series we observed deterioration of visual acuity despite recommended continuous treatment.

\section{CONCLUSION}

Autosomal dominant neovascular inflammatory vitreoretinopathy is a very serious progressive disease. Medical therapy for ADNIV has limited efficacy. Despite the aggressive treatment, we observed deterioration of visual acuity due to severe complications of the disease. The patients with ADNIV require constant ophthalmological care. Further investigations including immunology, physiology and genetics are necessary to better understand $\mathrm{AD}$ NIV pathogenesis.

\section{REFERENCES}

1. Michaelides M, Moore AT. Vitreous. In: Hoyt C, Taylor D. ed. Pediatric Ophthalmology and Strabismus. Elsevier, London 2012: 410-411.

2. Bassuk A, Yeh S, Wu S, et al. Structural Modeling of a Novel CAPN5 Mutation that Causes Uveitis and Neovascular Retinal Detachment. PLOS ONE . 2015; 10(4): e0122352, doi: 10.1371/journal. pone.0122352, indexed in Pubmed: 25856303.

3. Mahajan V, Skeie J, Bassuk A, et al. Calpain-5 Mutations Cause Autoimmune Uveitis, Retinal Neovascularization, and Photoreceptor Degeneration. PLoS Genetics. 2012; 8(10): e1003001, doi: 10.1371/ journal.pgen.1003001, indexed in Pubmed: 23055945.

4. Benett R, Folk JC, Kimura AE, et al. Autosomal Dominant Neovascular Inflammatory Vitreoretinopathy. BGSPlumX Metrics Ophthalmology. 1990; 97(9): 1125-1136.

5. MalaCards: Human Disease Database. https://www.malacards.org/.

6. Cham A, Bansal M, Banda HK, et al. Secondary glaucoma in CAPN5associated neovascular inflammatory vitreoretinopathy. Clin Ophthalmol . 2016; 10: 187-1197, doi: 10.2147/0PTH.S103324, indexed in Pubmed: 27390515.

7. Wert KJ, Skeie JM, Bassuk AG, et al. Functional validation of a human CAPN5 exome variant by lentiviral transduction into mouse retina. Human Molecular Genetics. 2013; 23(10): 2665-2677, doi: 10.1093/ $\mathrm{hmg} / \mathrm{ddt} 661$.

8. Vitreoretinopathy, neovascular inflammatory; VRNI. https://www. omim.org/entry/193235.

9. Mahajan VN, Folk JC, Fingert JH, et al. ARVO Annual Meeting. Genetic analysis and phenotypic staging of autosomal dominant neovascular inflammatory vitreoretinopathy. Invest Ophthalmol Visual Sci. 2011; 52(62): A175. 
10. Rowell HA, Bassuk AG, Mahajan VB, et al. Monozygotic twins with CAPN5 autosomal dominant neovascular inflammatory vitreoretinopathy. Clin Ophthalmol. 2012; 6: 2037 -2044, doi: 10.2147/OPTH. S40086, indexed in Pubmed: 23271883.

11. Wert K, Bassuk A, Wu WH, et al. CAPN5mutation in hereditary uveitis: the R243L mutation increases calpain catalytic activity and triggers intraocular inflammation in a mouse model. Human Molecular Genetics. 2015; 24(16): 4584-4598, doi: 10.1093/hmg/ddv189, indexed in Pubmed: 25994508.
12. Mahajan V, Lin J. Lymphocyte infiltration in CAPN5 autosomal dominant neovascular inflammatory vitreoretinopathy. Clin Ophthal. 2013; 1339, doi: 10.2147/opth.s46450, indexed in Pubmed: 23861576 ..

13. Tlucek P, Folk J, Orien J, et al. Inhibition of Neovascularization but Not Fibrosis With the Fluocinolone Acetonide Implant in Autosomal Dominant Neovascular Inflammatory Vitreoretinopathy. Arch Ophthalmol. 2012; 130(11): 1395, doi: 10.1001/archophthalmol.2012.1971, indexed in Pubmed: 22777573. 